\title{
Women kept up appearances and developed multiple coping strategies to deal with menopause
}

\author{
Kittell LA, Kernoff Mansfield P, Voda AM. Keeping up appearances: the basic social process of the menopausal transition. Qual \\ Health Res 1998 Sep;8:618-33.
}

\section{Question}

What are women's experiences of the menopausal transition and how do they interpret and respond to the changes they experience?

\section{Design}

Grounded theory.

\section{Setting}

USA.

\section{Patients}

61 women who were experiencing menopausal transition (menstrual bleeding changes, hot flashes, and change in feelings and emotions). Age range was 41-54 years and most were white, middle class, college educated, and employed outside the home.

\section{Methods}

Women were interviewed by telephone (23-110 min each with most lasting 40-45 $\mathrm{min}$ ) and asked to describe changes they were experiencing, how they felt about the changes, and ways in which the changes affected their lives. Changes could be related to bleeding, hot flashes, feelings and emotions, sleep, appearance, energy level, weight, appetite, digestion, sexual feelings, etc. Data were analysed, processes were identified, and a theory was developed.

\section{Main findings}

The basic core variable (social process) was described as keeping up appearances. Appearance encompassed all aspects of presentation of the self and perceptions of being a certain type of person. Women attempted to keep up appearances by concealing changes and by maintaining a sense of control in relation to physical and emotional changes. Women hid changes by keeping silent, keeping to themselves, detaching themselves, masking feelings or behaviours, avoiding discussions, diverting inquiries, and ignoring, minimising, or dismissing the effects of changes.

The unpredictability of the changes left women feeling as though they had no control over what was happening to them. They wanted control to avoid embarrassment and to be able to function with minimal disruption. The control was maintained through management strategies (increased self monitoring and self care, monitoring the environment and interactions with others, and making adjustments).

Women felt vigilant and anxious in situations that could potentially disclose their menopause status, and confident and secure when threat of disclosure was absent. Women sought help or felt frustrated in situations of potential social exposure and silent and detached when the threat of exposure was absent.

Women's feelings about, and responses to, changes varied over time and according to the characteristics of the change. If they felt the changes were normal and not disruptive, they felt secure and undisturbed. If they felt the changes were unexplained or disruptive, they became vigilant and used strategies to find out what was happening and how to minimise the disruption. With no control and high threat of social disclosure, women sought professional help.

\section{Conclusions}

When women experienced the menopausal transition they sought to regain control of the physical and emotional changes to keep up appearances. They gained control by concealing changes and developing multiple management strategies.

Source of funding: no external funding.

For correspondence: $\operatorname{Dr}$ L A Kittell, 137 Laurel Run, Portage, PA 15946, USA. Email ajlak@csrlink.net.

\section{Commentary}

The study presented by Kittell et al provides an intimate description of the needs and concerns of women experiencing menopausal transition and shows the individuality of the menopause experience. This research is timely given the current debate on hormone replacement therapy and medical management of menopause. Research that focuses on the feelings, behaviours, and daily challenges of women who are experiencing changes in their menstrual cycle has not previously been published.

Data were generated by a single telephone call to each participant, and the interviews were guided by a prepared interview schedule. The interview might not have provided the participants with adequate opportunity to reflect and articulate the most important aspects of living through menopausal change. Validity of the analysis process might have been enhanced if the women were invited to review the findings. However, illustration of the women's experiences using their words verbatim has provided an understanding of how the researchers arrived at their findings.

Two key concepts emerged from the data-concealment and control. During menopausal transition, the women described a desire to conceal the changes they were experiencing from others, and to maintain a sense of control over the social and functional aspects of their lives. From these concepts the researchers developed the core variable of keeping up appearances. The term appearance encompassed all aspects of the presentation of self.

This study shows how unpredictable life during the menopausal transition can be, and its findings should be made widely available to women in the community who often seek medical intervention only if they can no longer tolerate or control their symptoms. Johnson's extensive pre- vious research has shown that having accurate expectations reduces distress. ${ }^{1}$ Thus, the more women know about menopause, the less distress they should experience.

Through this study, the reader is sensitised to the experiences of women living through menopausal transition, and as such, the findings are not only important to nurses but also to the wider community. The study also shows that the broader cultural values of privatisation and self regulation influence women's interpretations of, and responses to, their menopausal experiences.

Debbie Kralik, RN, MN, MRCNA Research Associate Royal District Nursing Service Glenside, South Australia, Australia

1 Johnson JE, Fielder V,Jones L, et al. Self-regulation theory: applying theory to your practice. Pittsburg, PA: Oncology Nursing Press, Inc, 1997. 